\title{
Research on Gear CAD Design Based on UG Platform
}

\author{
Ting Liu \\ Jining Polytechnic, Jining, Shandong, China, 272037
}

Keywords: UG Platform; Gear; CAD Design; Design Optimization

\begin{abstract}
With the continuous development of computer technology, Chinese scientific level to further enhance the CAD / CAM technology gradually mature, and in the aerospace, construction, electronics, machinery, automobiles and other fields are widely used, and in the machinery industry, gear Transmission is one of the most common transmission methods, the effective transmission of sports and power parts, the successful completion of the commutation, change, speed, deceleration and other actions, which put the gear parts put forward higher requirements. This paper mainly analyzes and studies the UG platform, the significance of UG platform in gear CAD design and the gear CAD design analysis based on UG platform, which is helpful to strengthen the design of UG platform in gear CAD design. Application provides a reference.
\end{abstract}

\section{Introduction}

In all kinds of mechanical products, gears are one of the important parts, with an irreplaceable role. For a long time, CAD users are working to solve a problem, that is, if the gear design efficiency. Although the design process has gradually begun to use two-dimensional design method, but with the continuous development of CAD technology, in all aspects are gradually began to widely applied three-dimensional modeling method, even in most CAD software parts modeling has been widely used Three-dimensional modeling methods, such as Solidwork, Solidedge, Ides, Pro / E, UG and so on. In these CAD software, all include a variety of physical modeling methods, but if the gears in three-dimensional modeling and three-dimensional design using these software platforms, then not only need to spend a lot of time, and ultimately the formation of the design drawings are not necessarily Able to meet the relevant standards. In addition, the use of three-dimensional modeling to form two-dimensional graphics, the gear projection line problem is more prominent and Chinese gear mapping standards do not match, but increased the design difficulty. Therefore, based on UG platform gear CAD design, it is necessary to strengthen the research on the whole development step, and to take measures to solve the problems in the two-dimensional graphics generation, improve the gear CAD design level and quality, improve gear product design and Manufacturing accuracy, and thus meet the needs of various fields.

\section{The Overview of UG Platform}

UG is Unigrapgics NX short, is a kind of flattening engineering solutions, digital verification and modeling means, mainly used in product processing and product design process. UG as a computer aided design and computer aided manufacturing (CAD / CAM) system, has a very powerful function, to achieve a variety of complex modeling and physical construction [1]. Although in the early stages of development, it is mainly based on the workstation, but with the rapid development of PC hardware technology and rapid growth of individual users, gradually in the PC has been widely used in the mold industry, three-dimensional design has become one of the mainstream applications. In the product design and manufacturing process, if the shape is more complex and diverse, then the single piece of manufacturing and production of the relatively large degree of difficulty, the use of UG platform, in the design can be applied to composite modeling methods, the product intuitive manufacturing and thus to ensure its production quality. UG platform features include the following aspects:

The Entity Modeling Module. The solid modeling module can effectively combine the explicit 
geometric modeling and the constrained feature modeling, and use the remarkable composite modeling ability to design the various patch model and solid model with complex shapes, including the automatic shape modeling module and feature modeling module two parts [2].

User-Defined Feature Module. In the user-defined feature module, the main method is the interactive method. The constructed part feature is defined by the user. The timely parameterized entity model has been established and still can capture the parameter relationship.

Engineering Drawing Module. According to the established assembly model and the entity model information, the engineering drawing module can draw the parts drawing, the detail assembly drawing, the assembly drawing and the corresponding section diagram directly. In UG platform management, because the use of the Zhu model database, which means that the specific size of the drawings will be followed by changes in the model to make the appropriate adjustments, and thus ensure that the model size and drawing the same size [3].

Assembly Modeling Module. Assembly modeling module product development method is top-down, with a certain degree of synchronization, the completion of the entire structure of the assembly, parts positioning is very flexible, with each other to effectively cooperate. When the position and size of the parts are changed, the system will spontaneously reassemble, adjust the part position and size, and ensure its consistency.

Computer Aided Manufacturing Module. The computer-aided manufacturing module mainly contains the processing programming method. By applying this module, it can effectively process large parts of shaft parts. It is mainly used in multi-layer cutting process, which can remove the excess metal accurately.

\section{The Significance of Application of UG Platform in the Gear CAD Design}

Gear design is a form of precision machining, with a relatively high production efficiency, once the processing time has been reduced, it means that the cost has been reduced, the production period is significantly shorter. The use of CAD design, to design and manufacture of complex shapes, low roughness, high precision gear parts to meet the aerospace, watches, automobiles, machinery and other areas of demand [4].

Design drawings will directly determine the quality of gears, mold structure is reasonable and accurate, but also on the manufacture of gear products have a great impact on the quality. In the traditional design and manufacturing, is still the designer's experience, and therefore there are more problems, mainly in the following areas: First, the gear structure is more complex, if it is done using artificial design calculation and analysis process, then the time spent is relatively long, heavily dependent on the designer's experience; the second is if the design drawings are designed, it cannot be directly used in the NC processing, which led to extended production cycle, And also to a certain extent, the impact of gear manufacturing accuracy; Third, the traditional drawing tools need to spend a lot of time and effort, under normal circumstances will take about $75 \%$ of the design time, greatly reducing its design efficiency.

Through the UG platform for gear CAD design, in the design, calculation and analysis capabilities are improved at the same time, its design structure has also been significantly optimized, when the completion of the mold design, the design drawings can be automatically drawn out [5]. And through the introduction of parallel engineering technology in the CAD / CAM system, the design time is greatly shortened, in the mold CAM can directly apply the system design information, gear design, manufacturing integration, the final product quality has been further enhanced

\section{Gear CAD Design Analysis Based on the UG Platform}

Design Ideas. Gear CAD module includes three sub-modules, namely, bevel gear, helical gears, spur gears, in which the spur gear can be subdivided into variable gear and standard gear two types, the following figure shows the spur gear matrix. 


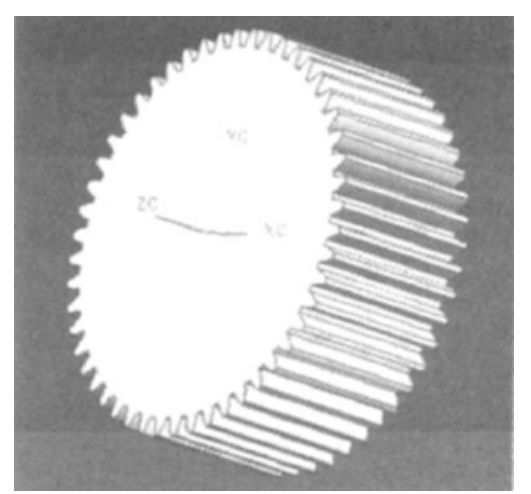

Figure 1 Schematic diagram of the spur gear base

The following is an involute spur gear, for example, the design of the calculation method to elaborate:

In the design of gear transmission, known conditions include the work load characteristics, service life, transmission ratio, speed, transmission torque or power. In the design calculation process, the first step is to select the corresponding parameters; the second step according to the simplified calculation method to determine the main gear size, and review. When the main geometry and parameters of the gears are determined, the gear structure can be designed. The third step is to determine the size and type of the gear structure; the fourth is to design the three-dimensional modeling; five is to generate two-dimensional engineering drawings; the other is to select the gears and the production of raw materials; [6].

In the process of developing the program, in order to facilitate the user to use, are used in the form of dialog box to select the gear in the structure type, modulus, number of teeth, transmission ratio and other major parameters, the following figure shows the gear parameters input box, including the number of teeth, surface modulus, pressure angle and many other parameters, in the development process, the user can directly set the parameters to complete the various types of gear design. When the design is completed, it needs to convert the data table into a file form, stored in the disk so that the program can run automatically find.

Three-Dimensional Modeling Method. As parametric graphics software, UG software in the three-dimensional modeling, not only can use the design data to complete the three-dimensional solid modeling, can also be gear accuracy level, gear thickness, tooth side clearance, tooth top high coefficient, Pressure angle, number of teeth, modulus and other related parameters directly into the dialog box for solid modeling. There are three types of gears that are commonly used: First, according to the relevant parameters in the gear, the tooth and tooth profile are generated and then stretched into a three-dimensional solid, which can be It can be directly seen as a tool to process the gear, the tank body can be removed directly after the formation of the tooth; the second is in accordance with the relevant parameters, the first generation of a gear in a single tooth, the tensile into a three-dimensional entity, you can rotate the copy according to the center of the circle; Third, in accordance with the relevant parameters, first in the plane will be all the teeth are generated, and then the overall stretch [7]. These three methods, whether it is the size of the file, or the production speed, there are significant differences, the first method of the smallest file, the fastest; the third method of the largest file, the slowest. Therefore, the first generation method is the best choice, the specific modeling steps of the method are:

1 , according to the gear thickness and the diameter of the top circle to generate the corresponding cylindrical solid, and thus the formation of simple tooth blank;

2 , according to the gear-related parameters and involute equation, generate involute cogging contour;

3, stretching the contour of the alveolar, the formation of three-dimensional entities, as the modulus cutter on the involute gear for processing;

4, the cylindrical axis as the involute cogging solid extension center, the number of array teeth;

5 , the use of subtraction boolean operation on the cylindrical entity, the stretched cogging entity minus, and finally the formation of spur gears. 
It is to be noted, however, that in the second step, the resulting involute cog is not the final involute, but only the spline formed by multiple keys on the involute, which Mainly due to a lot of CAD systems in the involute cannot be directly generated. Throughout the process, the points on the involute can be calculated using the involute equation, and the points are fitted through the spline curve, just the alveolar contours similar to the involute [8].

Solve the Tooth Projection Line Problem in the Two-Dimensional Figure. In the larger UG platform, two-dimensional drawings and three-dimensional model information are concentrated in a document. One of the three-dimensional model through the projection relationship, projection derived two-dimensional graphics, which can show that there is a direct link between the two. In the conventional mechanical parts, the two-dimensional graphics and three-dimensional model there is a good way of correlation between the model and the drawings can always be consistent. But in the gear design, if only the three-dimensional model directly to the formation of two-dimensional graphics, then the two-dimensional drawings will exist on all the gear teeth. In particular, in the side view, there will be all the visible lines and the crested lines on the $2 \mathrm{D}$ drawings, which do not correspond to the methods in the relevant drawing standards, and as long as the gear parts are included in the part drawing this problem occurs [9]. Therefore, there is a need for active development of solutions.

The solution is: in the development process, you can set up a separate layer, used to place the tooth three-dimensional entity, set it to A, follow-up gear wheel shape are on the basis of this layer. And then re-set a layer, used to place a specific three-dimensional entities (used to generate two-dimensional graphics), the resulting two-dimensional graphics to meet the relevant standard requirements, the layer is set to B. First in the B layer with a specific three-dimensional entities to generate two-dimensional graphics, you can hide it, and then in the process of developing the program, you can directly use the A layer of three-dimensional solid model. It should be noted that, in the generation of two-dimensional graphics, also need to hide the first layer.

\section{Conclusion}

The use of UG platform for gear CAD design, to achieve three-dimensional gear shape, compared to the single tooth and tooth in accordance with the overall stretch after the rotation copy of the method, not only can take up less disk space, graphics files, the amount of data is small, the program runs fast, while the use of multi-layer method, the gear tooth three-dimensional model of the formation of the mapping standards do not match the lines and the problem can be effectively resolved. In the gear CAD module development application UG development language, and in the main interface of UG plug, you can quickly generate gear two-dimensional drawings and unit modeling.

\section{References}

[1] Yang Ming. Based on ZG340-640 cast steel casting fork casting process CAD design[J]. Journal of Guizhou Normal University (Natural Science Edition), 2016, (04): 91-97.

[2] Mou Yinru. Computer Aided Design Based on Gear Parts on UGNX Platform [J]. Modern Manufacturing Technology \& Equipment, 2014, (06): 63-64.

[3] Wang Desheng, Si Yuanyuan, Zong Yadong. Multi-digital method and program development of experience coefficient graph or number table in gear design [J]. Journal of Henan Polytechnic University (Natural Science Edition), 2013, (06): 713-718.

[4] Kang Yanting, Qiu Zhixuan, Wang Dawei, Song Jianwei. CAD design of asynchronous starting permanent magnet synchronous submersible motor[J]. Explosion-proof motor, 2013, (06): 16-18.

[5] Zhou Lichao. Discussion on the design of locomotive transmission gear[J]. Electric locomotive and urban rail vehicle, 2013, (01): 64-68. 
[6] Hu Linlan, Yu Lingling. Gear design and three-dimensional modeling system based on VB and SolidWorks [J]. Mechanical Engineering \& Automation, 2012, (06): 34-36

[7] Liu Ping-ping, Qian Dan-hao. Three-dimensional accurate modeling of involute gear based on optimization design theory and UG platform[J]. Coal Mine Machinery, 2011, (09): 227-229.

[8] Zhang Jian-gang, Chi Cheng-zhong, Liu Xin-hui, Wang Ying. Preparation of chip punching die design system based on UG platform [J]. Die \& Mold Industry, 2011, (01): 13-16.

[9] Qin Jie, Xu Xiaoming, Zhao Yunsheng, Zhang Xiaolu. Application of Pro / E software in mechanical CAD design[J]. Equipment Manufacturing Technology, 2011, (01): 120-121. 